\title{
Perfil nutricional de mães de prematuros e avaliação de diferentes fatores de risco e carências nutricionais relacionadas ao parto prematuro - revisão sistemática
}

\author{
Nutritional profile of mothers of premature infantis and evaluation of different risk \\ factors and nutritional deficiencies related to preterm birth - systematic review
}

José Francisco Alves de Andrade', Henrique Custódio da Silva', Thiago Brito Xavier', Sherlon Elvis Pinto Raio', Monique de Almeida Hingel de Andrade², Mauro de Souza Pantoja'

\begin{abstract}
Resumo Objetivo: Apresentar e discutir os achados da literatura referentes ao perfil nutricional de mães de prematuros na população em geral e identificar os diferentes fatores de risco e carências nutricionais relacionadas ao parto prematuro. Método: Os dados foram coletados mediante revisão sistemática utilizando os descritores gestação, estado nutricional, recém-nascido prematuro, tomando como base artigos levantados dos últimos 5 anos publicados no Scientific Electronic Library (Scielo), Lilacs, MedLine e Pubmed. Como critérios de inclusão foram estabelecidos artigos originais e de revisão publicados na íntegra na forma on-line nos idiomas português, espanhol e inglês no período de 2012 a 2017, utilizando os descritores supracitados nos respectivos idiomas. Como critério de exclusão foram estabelecidos artigos repetitivos nas bases de dados, além de artigos não pertinentes à busca nos descritores definidos. Resultados: Foram coletados 85 artigos científicos, nos quais 70 destes se encontraram dentro dos critérios de exclusão e 15 trabalhos dentro dos critérios de inclusão. Conclusão: O perfil das mães de prematuros é influenciado pelas condições socioeconômicas e sanitárias. A maioria apresentou taxas de zinco eritrocitário inferior aos das mães de bebês a termo. A ingestão excessiva de proteínas favorece a incidência de prematuridade. Mães obesas ou com baixo peso contribuem para a morbimortalidade neonatal e consequentemente prematuridade. Desta forma, a avaliação do consumo alimentar, da suplementação e do estado nutricional durante o processo gravídico são importantes para detectar déficits nutricionais e hábitos inadequados, com vista a adequar as necessidades de nutrientes nesse período e a evitar alterações no estado nutricional, permitindo um prognóstico favorável à saúde da mãe e filho.
\end{abstract}

Descritores: gestação; estado nutricional; recém nascido prematuros.

Summary Purpose: Introduce and discuss the literature findings in reference of mothers' and preterms' nutricional profile in the general population and to identify the different risk factors and nutricional deficiencies related to preterm delivery. Methods: The data were colatated by reviewing the literature using the descriptors gestation, nutricional status, premature newborn, based on articles from the last 5 years published in Scielo, Lilacs, Medline and Pubmed. As criteria inclusion, original and review articles were published online in Portuguese, Spanish and English during the period from 2013 to 2018, using the descriptors mentioned above. As exclusion criteria repetitive articles were established in the database, as well as artucles not pertinent to the search in the defined descriptors. Results: 85 articles were collected in which 70 were in the exclusion criteria and 15 in the inclusion criteria. Conclusion: The pregnant woman needs an adequate diet with proteins, vitamins and minerals because it needs to generate energy for itself and for the fetus that is developing new tissues and organs. In addition, food consumption, nutritional status and supplementation should be evaluated during the pregnancy period.

Keywords: nutritional status; infant premature; preganancy.

${ }^{1}$ Universidade Estadual do Pará - UEPA, Belém, PA, Brasil

${ }^{2}$ Universidade Metropolitana da Amazônia - FAMAZ, Belém, PA, Brasil

Fonte de financiamento: Nenhuma.

Conflito de interesses: Os autores declaram não haver conflitos de interesse.

Recebido: Agosto 10, 2018

Aceito: Outubro 01, 2018

Trabalho realizado no Laboratório de Cirurgia Experimental - LCE, Curso de Mestrado de Cirurgia e Pesquisa Experimental, Universidade Estadual do Pará UEPA, Belém, Pará, Brasil.

Copyright Andrade et al. Este é um artigo publicado em acesso aberto (Open Access) sob a licença Creative Commons Attribution, que permite uso, distribuição e reprodução em qualquer meio, sem restrições desde que o trabalho original seja corretamente citado. 


\section{Introdução}

O prognóstico da gestação, conforme estudos experimentais e clínicos tem relação direta com o estado nutricional materno antes e durante a gestação em relação ao desenvolvimento do feto e saúde do neonato, assim como a capacidade materna para a lactação ${ }^{1}$. O período gestacional é dividido em duas fases: uma fase materna, na qual as necessidades nutricionais adequadas são importantes para os ajustes fisiológicos do organismo materno como do desenvolvimento fetal e, uma fase fetal ( $2^{a}$ metade da gestação), onde ocorre extrema necessidade de nutrientes e vitaminas para o perfeito crescimento fetal ${ }^{2}$.

A avaliação nutricional individualizada no início do pré-natal é importante para estabelecer as necessidades de nutrientes nesse período e deve ser realizada continuamente ao longo da gravidez ${ }^{2,3}$. Desse modo, o conhecer do perfil nutricional de uma população de gestantes possibilita o melhor planejamento de ações em saúde que visam melhorar a qualidade da assistência pré-natal e prevenir agravos nutricionais no período neonatal.

Os indicadores antropométricos indispensáveis para o diagnóstico nutricional da gestante segundo a Organização Mundial da Saúde são as medidas de peso e estatura isoladas e suas combinações, como adequação percentual de peso por estatura (P/E), e o índice de massa corporal (IMC) ${ }^{3}$. A avaliação dietética da gestante por meio de inquéritos alimentares favorece a análise e avaliação do consumo alimentar praticado, já que os hábitos alimentares dos diferentes grupos sociais são determinados pela capacidade de acesso aos alimentos, pelas suas crenças religiosas e pelos seus valores culturais ${ }^{4}$. Vale ressaltar que, como a alimentação materna influencia diretamente no desenvolvimento e crescimento fetal, o peso do neonato correlaciona-se com a probabilidade de sobrevida neonatal; tendo em vista que o peso inadequado ao nascimento indica risco elevado de morbidade e mortalidade no primeiro ano de vida ${ }^{5}$.

As necessidades nutricionais maternas devem ser consideradas e atendidas, levando-se em conta que a amamentação é um estado fisiológico com intenso processo metabólico. Ocorre com a produção láctea aumento na demanda energética e nutricional para garantir que as reservas maternas não sejam depletadas em benefício deste processo ${ }^{4,5}$. Além de que, as deficiências nutricionais da nutriz podem contribuir para a manutenção de baixas reservas de alguns nutrientes nos lactentes, aumentando as chances para o desenvolvimento de carências nutricionais nos primeiros anos de vida ${ }^{6}$.

Além disso, na Região Norte e especialmente no Pará, por suas peculiaridades geográficas, demográficas, socioeconômicas e políticas, tem-se observado um elevado número de gestantes desenvolvendo gestações de alto risco, o que oferece perigo tanto materno, quanto fetal, necessitando de atendimento especializado e de referência ${ }^{7}$.

A Organização Mundial da Saúde (OMS) constata que o estado de nutrição materno pré-gestacional, gestacional e o ganho de peso inadequado nesse período é fator agravante das causas de mortalidade materna e influenciam nas condições perinatais ${ }^{3}$. Desta forma a avaliação do estado nutricional da gestante, bem como a avaliação do consumo dietético durante esse período é de fundamental importância para futuras intervenções nutricionais e desenvolvimento correto da gestação minimizando os riscos para o concepto e gestantes.

\section{Objetivo}

Realizar revisão sistemática sobre o perfil nutricional de mães de prematuros e avaliar diferentes fatores de risco e carências nutricionais relacionadas ao parto prematuro.

\section{Método}

Realizada a revisão sistemática com seleção das publicações mais relevantes sobre o perfil nutricional de mães de prematuros e avaliar diferentes fatores de risco e carências nutricionais relacionadas ao parto prematuro utilizando as bases de dados do Scientific Electronic Library (Scielo), Lilacs, MedLine e Pubmed em português, espanhol e inglês. Usou-se como critério de inclusão artigos relacionados ao tema publicados entre os anos de 2013-2018, e como critério de exclusão artigos que não apresentavam relevância sobre o tema e/ou publicados fora do espectro de data estabelecido.

Foram pesquisados 85 artigos científicos sendo que 70 deles não encontravam-se dentro dos critérios de inclusão. 


\section{Resultados}

Finalizada a seleção dos artigos, realizou-se a organização sistemática dos mesmos a fim de orientar sobre seus t ítulos, objetivos, ano, metodologia e periódico(quadro 1)

Quadro 1. Síntese dos artigos incluídos na revisão segundo título, objetivo, ano, metodologia e periódico

\begin{tabular}{|c|c|c|c|c|}
\hline TÍTULO & OBJETIVO & ANO & METODOLOGIA & PERIÓDICO \\
\hline $\begin{array}{l}\text { Associação entre qualidade do } \\
\text { pré-natal e baixo peso ao nascer } \\
\text { em uma Instituição hospitalar em } \\
\text { Feira de Santana }\end{array}$ & $\begin{array}{l}\text { Medir a associação entre } \\
\text { qualidade do pré natal e baixo } \\
\text { peso ao nascer em usuários de um } \\
\text { hospital de Feira de Santana/BA. }\end{array}$ & 2018 & Caso Controle & $\begin{array}{l}\text { Revista de } \\
\text { epidemiologia } \\
\text { e controle de } \\
\text { infecção do } \\
\text { Hospital Santa } \\
\text { Cruz }\end{array}$ \\
\hline $\begin{array}{l}\text { Maternal and neonatal factores } \\
\text { related to prematurity }\end{array}$ & $\begin{array}{l}\text { Identificar fatores maternos } \\
\text { e neonatais associados com a } \\
\text { prematuridade no município de } \\
\text { Porto Alegre. }\end{array}$ & 2016 & Caso controle & $\begin{array}{l}\text { Revista da escola } \\
\text { de enfermagem } \\
\text { USP }\end{array}$ \\
\hline $\begin{array}{l}\text { Fatores nutricionais e sociais de } \\
\text { importância para o resultado } \\
\text { da gestação, em mulheres em } \\
\text { acompanhamento na rede de } \\
\text { atenção primária de Juiz de Fora }\end{array}$ & $\begin{array}{l}\text { Avaliar o estado nutricional e } \\
\text { de saúde, analisar o consumo } \\
\text { alimentar e investigar o perfil } \\
\text { socioeconômico de gestantes } \\
\text { atendidas em unidades de saúde } \\
\text { de Juiz de Fora MG. }\end{array}$ & 2014 & Quantitativa & $\begin{array}{l}\text { Revista médica de } \\
\text { MG }\end{array}$ \\
\hline $\begin{array}{l}\text { Perfil epidemiológico das } \\
\text { gestantes atendidas em um centro } \\
\text { de referência em pré-natal de alto } \\
\text { risco }\end{array}$ & $\begin{array}{l}\text { Conhecer o perfil das gestantes } \\
\text { atendidas em um Centro } \\
\text { de referência em pré-natal } \\
\text { de alto risco, no município } \\
\text { de Santarém, período de } \\
\text { setembro de } 2010 \text { a setembro } \\
\text { de } 2011 \text {, intencionando-se } \\
\text { identificar fatores de risco, que, } \\
\text { possivelmente, contribuíram } \\
\text { para o desenvolvimento de uma } \\
\text { gestação de alto risco }\end{array}$ & 2014 & Quantitativo & $\begin{array}{l}\text { Revista Paraense } \\
\text { de Medicina }\end{array}$ \\
\hline $\begin{array}{l}\text { Perfil epidemiológico e nutricional } \\
\text { de adolescentes grávidas } \\
\text { internadas em um hospital de } \\
\text { referência do estado do pará }\end{array}$ & $\begin{array}{l}\text { Identificar o perfil epidemiológico } \\
\text { e situação nutricional de gestantes } \\
\text { adolescentes internadas em um } \\
\text { hospital de referência do Estado } \\
\text { do Pará. }\end{array}$ & 2014 & Quantitativo & $\begin{array}{l}\text { Revista Paraense } \\
\text { de Medicina }\end{array}$ \\
\hline $\begin{array}{l}\text { Caracterização nutricional de } \\
\text { nutrizes na segunda etapa do } \\
\text { método canguru de um hospital } \\
\text { amigo da criança. }\end{array}$ & $\begin{array}{l}\text { Caracterizar nutricionalmente } \\
\text { as nutrizes na segunda etapa do } \\
\text { método canguru de um Hospital } \\
\text { Amigo da Criança. }\end{array}$ & 2015 & $\begin{array}{l}\text { Quantitativo } \\
\text { descritivo }\end{array}$ & $\begin{array}{l}\text { Revista Paraense } \\
\text { de Medicina }\end{array}$ \\
\hline $\begin{array}{l}\text { Fatores nutricionais e sociais de } \\
\text { importância para o resultado } \\
\text { da gestação em mulheres em } \\
\text { acompanhamento na rede de } \\
\text { atenção primária de Juiz de Fora. }\end{array}$ & $\begin{array}{l}\text { Avaliar o estado nutricional e } \\
\text { de saúde, analisar o consumo } \\
\text { alimentar e investigar o perfil } \\
\text { socioeconômico de gestantes } \\
\text { atndidas em Unidades de Saúde } \\
\text { de Juiz de Fora (MG). }\end{array}$ & 2015 & $\begin{array}{l}\text { Observacional } \\
\text { descritivo }\end{array}$ & Revista Med MG \\
\hline $\begin{array}{l}\text { Suplementação na infância e } \\
\text { a prevenção da carência de } \\
\text { micronutrientes. }\end{array}$ & $\begin{array}{l}\text { Delinear sobre o aporte } \\
\text { complementar dos principais } \\
\text { nutrientes preconizados pela } \\
\text { Sociedade Brasileira de Pediatria, } \\
\text { os quais as vitaminas A, D e K, } \\
\text { ferro e zinco, apontando seus } \\
\text { benefícios para a prevenção } \\
\text { de doenças e sua efetividade } \\
\text { para garantir o crescimento e o } \\
\text { desenvolvimento saudável das } \\
\text { crianças. }\end{array}$ & 2017 & $\begin{array}{l}\text { Revisão } \\
\text { bibliográfica }\end{array}$ & $\begin{array}{l}\text { Revista médica } \\
\text { Saúde Brasília }\end{array}$ \\
\hline
\end{tabular}


Quadro 1. Continuação...

\begin{tabular}{|c|c|c|c|c|}
\hline TÍTULO & OBJETIVO & ANO & METODOLOGIA & PERIÓDICO \\
\hline $\begin{array}{l}\text { Estado nutricional relativo ao } \\
\text { ferro e zinco de mães de recém- } \\
\text { nascidos de risco. }\end{array}$ & $\begin{array}{l}\text { Avaliar o estado nutricional } \\
\text { relativo ao ferro e zinco em mães } \\
\text { de bebês de risco. }\end{array}$ & 2014 & Corte transversal & Nutrire \\
\hline $\begin{array}{l}\text { Perfil nutricional de gestantes } \\
\text { atendidas em uma unidade básica } \\
\text { de saúde. }\end{array}$ & $\begin{array}{l}\text { Avaliar o perfil nutricional de } \\
\text { gestantes atendidas em uma } \\
\text { Unidade Básica de Saúde. }\end{array}$ & 2014 & $\begin{array}{l}\text { Observacional } \\
\text { transversal com } \\
\text { abordagem } \\
\text { Quali-quantitativa }\end{array}$ & Cinergis \\
\hline $\begin{array}{l}\text { Perfil de mães e o desfecho do } \\
\text { nascimento prematuro ou a termo }\end{array}$ & $\begin{array}{l}\text { Identificar o perfil de mães de } \\
\text { bebês prematuros e a termo para o } \\
\text { desfecho do nascimento. }\end{array}$ & 2015 & Quantitativa & $\begin{array}{l}\text { Revista UFPR } \\
\text { COGITARE }\end{array}$ \\
\hline $\begin{array}{l}\text { Estado nutricional relativo } \\
\text { ao ferro e zinco de mães de } \\
\text { recém-nascidos }\end{array}$ & $\begin{array}{l}\text { Avaliar o estado nutricional } \\
\text { relativo ao ferro e zinco em mães } \\
\text { de bebês de risco. }\end{array}$ & 2014 & Quantitativa & Nutrire \\
\hline $\begin{array}{l}\text { Preterm and late preterm } \\
\text { infants: their differences and } \\
\text { breastfeeding. }\end{array}$ & $\begin{array}{l}\text { To verify the differences between } \\
\text { preterm and late preterm infants } \\
\text { in relation to the duration of } \\
\text { breastfeeding and exclusive } \\
\text { breastfeeding causes and } \\
\text { consequences of early weaning. }\end{array}$ & 2015 & $\begin{array}{l}\text { Exploratório e } \\
\text { longitudinal }\end{array}$ & Revista CEFAC \\
\hline $\begin{array}{l}\text { Perfil Nutricional de Gestantes } \\
\text { Atendidas por Duas Unidades } \\
\text { Básicas de Saúde de Londrina-PR. }\end{array}$ & $\begin{array}{l}\text { Verificar o perfil nutricional de } \\
\text { gestantes atendidas por Unidades } \\
\text { Básicas de Saúde Municipais. }\end{array}$ & 2015 & Transversal & $\begin{array}{l}\text { Journal of Health } \\
\text { Sciences }\end{array}$ \\
\hline $\begin{array}{l}\text { Perfil nutricional materno e estado } \\
\text { nutricional neonatal, na cidade de } \\
\text { Pará de Minas - MG }\end{array}$ & $\begin{array}{l}\text { Avaliar a influência do estado } \\
\text { nutricional materno no peso ao } \\
\text { nascer de neonatos. }\end{array}$ & 2013 & Retrospectivo & $\begin{array}{l}\text { Revista médica } \\
\text { MG }\end{array}$ \\
\hline
\end{tabular}

\section{Discussão}

Vários estudos, conforme a pesquisa da literatura, procuraram estabelecer as relações entre o perfil nutricional materno e as condições clínicas e nutricionais do feto e chegaram a conclusões contraditórias.

A gestação é um período de maior demanda nutricional do ciclo de vida da mulher, pelo fato de envolver rápida divisão celular e desenvolvimento de novos tecidos e órgãos ${ }^{2}$. Dessa forma a ingesta de valores nutricionais adequados é de extrema importância para o prognóstico da gestação ${ }^{1}$.

A dieta, no primeiro trimestre, é importante para o desenvolvimento e diferenciação dos diversos órgãos fetais. Já nos trimestres subsequentes, a dieta está mais envolvida com a otimização do crescimento e do desenvolvimento cerebral do feto ${ }^{8}$. O perfil das mães de prematuros e a caracterização dos nascidos-vivos são influenciados pelas condições sócio-econômicas e sanitárias da localidade em questão onde ocorrem a gestação e o nascimento ${ }^{9}$. Estudos epidemiológicos demonstram que o maior risco para complicações gestacionais está relacionado à obesidade, embora o baixo peso também aumente os riscos de desfechos desfavoráveis para a mãe e, principalmente, para o concepto. Entre esses desfechos podem ser citados diabetes e hipertensão maternas, macrossomia, sofrimento fetal, trabalho de parto prolongado, parto cirúrgico, restrição de crescimento intrauterino e prematuridade 9 .

A inadequação do estado antropométrico materno, tanto pré-gestacional quanto gestacional, se constitui um problema de saúde pública, pois favorece o desenvolvimento de intercorrências gestacionais e influencia nas condições de saúde do feto e na saúde materna no período pós-parto ${ }^{9}$.

Crianças que nascem com peso menor que $2500 \mathrm{~g}$ (baixo peso ao nascer) estão sob alto risco de morrer ou adoecer. Gestantes adolescentes, em fase de crescimento, têm seus recém-nascidos com média de peso inferior em 150 - 200g quando comparados com neonatos de mães adultas ${ }^{10}$. Gestantes com ganho de peso insuficiente apresentam maiores riscos de gerarem recém-nascidos com peso inadequado, podendo comprometer o crescimento pós-natal, com maior risco de morbidade no primeiro ano de vida ${ }^{9,11}$ Outros indicadores do estado nutricional, como o consumo alimentar, também interferem diretamente sobre o resultado do concepto. Quando há consumo inadequado de nutrientes pela mãe, deve-se considerar o período 
da gestação em que ocorre, o quão grave é essa inadequação e por quanto tempo persiste ${ }^{10}$. Gestantes que apresentam uma reserva inadequada de nutrientes, aliada a uma ingestão dietética insufi ciente, poderão ter um comprometimento do crescimento fetal e, conseqüentemente, do peso ao nascer.

$\mathrm{O}$ aumento da necessidade de vitaminas e minerais no segundo e terceiro trimestres da gestação é acompanhado pelo correspondente aumento do apetite no mesmo período, o que torna, usualmente, desnecessária a suplementação desses nutrientes quando a gestante se alimenta adequadamente ${ }^{11}$. Estudos recentes sobre a necessidade de suplementação de zinco na gravidez não demonstraram associação entre o seu uso e a diminuição da incidência de baixo peso ao nascimento ou de complicações como prematuridade, hipertensão, amnionite e infecção pós-parto. ${ }^{12}$ Recomenda-se a suplementação de cálcio em gestantes que se alimentam pouco, que não toleram laticínios ou que usem medicamentos que inibam a absorção do cálcio (heparina, prednisona) ${ }^{12}$. A suplementação desnecessária de nutrientes pode, inclusive, implicar alguma toxicidade para o feto, causando abortamento, mal formações congênitas, parto prematuro ${ }^{11,12}$.

A gestação pode atuar como desencadeante da obesidade ou como agravante, quando esta for preexistente, com várias consequências adversas para as mulheres e seus bebês ${ }^{13}$. Para o concepto, as taxas de malformações fetais e de defeitos do tubo neural - mesmo naquelas que recebem suplementação de ácido fólico nas doses recomendadas - são mais altas. Os riscos de óbito e macrossomia fetais são significativamente mais elevados entre as mulheres com peso acima da média, por motivos ainda desconhecidos. O excesso de tecido adiposo materno parece ainda ser capaz de comprometer a programação metabólica fetal, predispondo os filhos de mulheres obesas a serem futuros obesos e diabéticos ${ }^{14}$.

Em relação aos macronutrientes não há vantagens em ingerir grande quantidade de proteína durante a gravidez/lactação, havendo, inclusive, indícios de que a ingestão excessiva de proteína nessa condição aumenta a incidência de prematuridade ${ }^{15}$. A média dos valores encontrados recomendados para o período gestacional em relação à energia, ferro e zinco na grande maioria dos estudos estavam abaixo dos valores recomendados, o que pode ser atribuído à dificuldade no atendimento dietético diante da elevação nas necessidades nutricionais para esse período. É importante ressaltar que estudos sobre a composição da dieta habitual de gestantes ainda são escassos e, geralmente, mostram resultados conflitantes. Mães de bebês prematuros apresentaram valores de ZnER (fração de zinco eritrocitário) inferiores àquelas mães de bebês a termo. A carência de ferro apresenta elevada prevalência mundial e, entre os segmentos populacionais mais vulneráveis ao problema, estão as mulheres no período reprodutivo, particularmente durante a gestação, e as crianças nos primeiros anos de vida, em função do aumento das necessidades desse mineral, decorrente da rápida expansão de massa celular vermelha e do crescimento acentuado dos tecidos nessas fases da vida ${ }^{5}$.

Estima-se que cerca de $60 \%$ das gestantes apresentem anemia, e mulheres no ciclo grávido-puerperal, mesmo após suplementação com doses de ferro recomendadas para o período gestacional, apresentam altos índices de anemia ferropriva. Note-se que esta deficiência pode estar associada ao aumento de mortalidade materna e perinatal, à prematuridade, ao baixo peso ao nascer (BPN) e à morbidade do infante 5 .

A reserva inadequada de nutrientes, aliada a uma ingestão dietética insuficiente, poderão levar ao comprometimento do crescimento fetal e, consequentemente, do peso ao nascer. Os riscos de óbito e macrossomia fetais são significativamente mais elevados entre as mulheres com peso acima da média, por motivos ainda desconhecidos. A ingestão excessiva de proteína aumenta a incidência de prematuridade.

O baixo peso materno e as carências específicas de micronutrientes podem resultar em anemia e carência de vitamina A, além de baixo peso do feto ao nascer, que é considerado um dos fatores que mais contribuem para a morbimortalidade neonatal e prematuridade.

Desta forma, a avaliação do consumo alimentar, da suplementação e do estado nutricional durante o processo gravídico são importantes para detectar déficits nutricionais e hábitos inadequados, com vista a adequar as necessidades de nutrientes nesse período e a evitar alterações no estado nutricional, permitindo um prognóstico favorável à saúde da mãe e filho.

\section{Agradecimentos}

Agradecemos ao Mestrado em Cirurgia Experimental da Universidade Estadual do Pará, na pessoa do Professor Dr. Marcus Henriques Brito. E ao Professor Dr. Mauro de Souza por suas contribuições em nossa formação. 


\section{Referências}

1. Coelho JMF, Galvão CR, Rodrigues RM, Carvalho SS, Santos BM, Miranda SS, et al. Asociación entre calidad del pre-natal y bajo peso al nascer en una institución hospitalaria en Feira de Santana/Ba. Rev Epidemiol Control Infec. 2018;8(2):129-35. http://dx.doi.org/10.17058/ reci.v1i2.10406.

2. Oliveira LL, Gonçalves AC, Costa JSD, Bonilha AL. Maternal and neonatal factors related to prematurity. Rev Esc Enferm USP. 2016;50(3):382-9. http://dx.doi.org/10.1590/S0080-623420160000400002. PMid:27556707.

3. Brasil. Ministério da saúde. Brasil incentiva ações e campanhas para garantir pré-natal a gestantes [Internet]. 2017 [citado em 2018 jan 19]. Disponível em: http://www.brasil.gov.br/sobre/saude/maternidade/gestacao/a-importancia-do-pre-natal

4. Ribeiro ECD, Moraes PMO, Moraes LP. Caracterização nutricional de nutrizes na segunda etapa do método canguru de um hospital amigo da criança. Rev Para Med = Rev Para Med. 2015;29(3):21-8.

5. Anjos JCS, Pereira RR, Ferreira PRC, Mesquita TBP, Picanço Jr OM. Perfil epidemiológico das gestantes atendidas em um centro de referência em pré-natal de alto risco. Rev Para Med = Rev Para Med. 2014;28(2):23-33.

6. Moraes LP, Moraes PMO, Ribeiro ECDR. Perfil epidemiológico e nutricional de adolescentes grávidas internadas em um hospital de referência do estado do Pará. Rev Para Med = Rev Para Med. 2014;28(4):49-56.

7. Andrade BD, Silva ACP, Santos MTM, Campos T, Luquetti SCPD. Fatores nutricionais e sociais de importância para o resultado da gestação em mulheres em acompanhamento na rede de atenção primária de Juiz de Fora. Rev Med MG. 2015;25(3):344-52.

8. Vaz MA, Oliveira GG, Pinheiro MS, Medeiros EEF. Suplementação na infância e a prevenção da carência de micronutrientes. Rev Med Saúde Brasilia. 2017;6(1):116-31.

9. Cavalcante RMS, Almeida LKL, Silva Na EA, Moita No JM, Marreiro DN, Nogueira NN. Estado nutricional relativo ao ferro e zinco de mães de recém-nascidos de risco. Nutrire. 2014;39(2):187-95. http://dx.doi.org/10.4322/nutrire.2014.017.

10. Lima RR, Molz P, Pereira CS. Perfil nutricional de gestantes atendidas em uma unidade básica de saúde. Cinergis. 2014;15(2):98-102. http:// dx.doi.org/10.17058/cinergis.v15i2.5134.

11. Marcuartú AC, Malveira SS. Perfil de recém-nascidos prematuros de muito baixo peso internados em unidade de cuidados intensivos neonatais. RBCS. 2016;21(1):5-10. http://dx.doi.org/10.4034/RBCS.2017.21.01.01.

12. Silva WF, Guedes ZCF. Preterm and late preterm infants: their differences and breastfeeding. Rev CEFAC. 2015;17(4):1232-40. http://dx.doi. org/10.1590/1982-0216201517417514.

13. Eleutério BM, Araújo GLO, Silveira LP, Anastácio LR. Perfil nutricional materno e estudo nutricional neonatal, na cidade de Pará de Minas-MG. Rev Med MG. 2013;23(3):311-17.

14. Amorin SMRF. Perfil nutricional de gestantes atendidas por duas unidades básicas de saúde de Londrina-PR. J Health Sci (Sarajevo). 2015;10(2):75-81.

15. Teixeira GA, Carvalho JBL, Rocha BG, Pereira SA, Enders BC. Perfil de mães e o desfecho do nascimento prematuro ou a termo. Cogitare Enfer. 2018;1(23):e51409. http://dx.doi.org/10.5380/ce.v23i1.51409. 


\section{Autor correspondente}

Thiago Brito Xavier

Universidade Estadual do Pará - UEPA, Instituto de Ciências da Saúde, Faculdade de Medicina, Mestrado de Cirurgia e Pesquisa Experimental - CIPE

Tv. Ezeriel Mônico de Matos, 43, Guamá

CEP 66075-225, Belém, PA, Brasil

Tel.: (91) 3349-6820 / (91) 99822-5094

E-mail: thiagohujbb@gmail.com

\section{Informação sobre os autores}

JFAA é médico, especialista em Pediatria e em Medicina Intensiva Neonatal pela Fundação Santa Casa de Misericórdia do Pará.

HCS é médico, especialista em Cardiologia pelo Conselho Regional de Medicina do Estado do Pará; atividade judicante do Conselho Regional Medicina do Pará.

TBX é odontólogo, especialista em Bucomaxilofacial pelo Hospital Universitário João de Barros Barreto.

SEPR possui graduação em Medicina pelo Centro Universitário do Estado do Pará.

MAHA possui graduação em Enfermagem e Obstetrícia pela Universidade Federal do Rio de Janeiro (UFRJ); graduanda em Medicina pela Faculdade Metropolitana da Amazônia (FAMAZ).

MSP possui graduação em Medicina pela Universidade Federal do Pará (UFPA); mestrado em Cirurgia pela Universidade Estadual de Campinas (UNICAMP); doutorado em Cirurgia pela Universidade Estadual de Campinas (UNICAMP); coordenador clínico da Nutrir Prestadora de Serviços Médicos Ltda.; coordenador da Equipe Multidisciplinar de Terapia Nutricional (EMTN); professor adjunto IV da Universidade Estadual do Pará (UEPA); especialista em Terapia Nutricional Enteral e Parenteral pela Sociedade Brasileira de Nutrição Parenteral; especialista em Terapia Nutricional e Nutrição Clínica pelo Grupo de Apoio de Nutrição Enteral e Parenteral (GANEP) da Faculdade Anhembi Morumbi.

\section{Contribuição dos autores}

JFAA foi responsável pela formatação e edição de manuscrito, atualização literária e edição para submissão; HCS foi responsável pela edição de manuscrito e atualização literária; TBX realizou levantamento bibliográfico, atualização literária e edição para submissão; SEPR fez levantamento bibliográfico e atualização literária; MAHA fez edição de manuscrito e atualização literária; MSP fez atualização literária, revisão e críticas. 